\title{
Status of MARS Electromagnetic Physics *
}

\author{
O. E. Krivosheev and N. V. Mokhov \\ Fermi National Accelerator Laboratory \\ P.O. Box 500, Batavia, Illinois 60510, USA
}

August 11, 2000

\begin{abstract}
New modules for simulating electromagnetic showers at energies from $1 \mathrm{keV}$ to a multi-TeV region have been developed and implemented into the MARS code. The entire shower and several "non-standard" processes essential at high energies are treated either exclusively or inclusively, according to the user's choice. Results of calculations are in a good agreement with data.
\end{abstract}

${ }^{*}$ Presented Paper at the Monte Carlo 2000 International Conference, Lisbon, Portugal, October 23-26, 2000 


\section{Introduction}

Developments in the MARS code [1] cover physics of hadron and lepton interactions from $100 \mathrm{TeV}$ down to a fraction of an electron volt for neutrons and to about $100 \mathrm{keV}$ for all other particles. Simulation of electromagnetic showers (EMS), although improved over the years, is still based —in the physics part—on the use of the AEGIS modules [2], which implies an inclusive approach and a simplified process description below a few $\mathrm{MeV}$. This is quite acceptable for the majority of energy deposition and radiation applications, but in some cases a full analog simulation and a detailed physics description in the low energy domain are needed. New modules have recently been developed and implemented into MARS, as described in this paper. The main focus is given to electron and photon interactions in arbitrary composite solid, liquid and gaseous materials at low energies ( $1 \mathrm{keV}$ to a few $\mathrm{MeV}$ ). The high energy part is also completely re-built. A generic polarized photon transport algorithm is implemented. The entire shower, and such processes as emission of synchrotron photons, photohadron production, $\gamma Z \rightarrow \mu^{+} \mu^{-}$and $e^{+} e^{-} \rightarrow \mu^{+} \mu^{-}$, can be treated-in the spirit of the MARS framework-either exclusively or inclusively with corresponding statistical weights. The choice of method is left for the user to decide.

\section{Photon Physics}

Simulation of photon physics is based upon data from the EPDL library [3]. MARS stores the evaluated data, pre-compiled for fast interpolation for all 100 elements over a uniform, material- and element-independent, energy scale. The material is considered as an independent mixture of constituents. Total cross-sections and mean free paths are calculated, together with branching probabilities from these tables, as weighted sums over the elements in the mixture for four main processes: $e^{+} e^{-}$pair production, photoelectric effect, Compton scattering and coherent scattering. Above $1 \mathrm{GeV}$, the photoelectric effect and coherent scattering cross-sections are assumed to be zero and high energy approximations are used for Compton scattering and $e^{+} e^{-}$ pair production.

In the course of simulations - after sampling the process and the element- the particle phase coordinates are generated and stored on the stack for further processing. Compton scattering is simulated from the Klein-Nishina formula with the scattering function correction due to electron binding to the atom, essential at low energies $\omega \leq 1 \mathrm{MeV}$. Coherent scattering is described in a form-factor approach, with data from EPDL. The scattering angle is sampled from the form-factor with the Rayleigh formula used to accept the sampled angle with a $\sim 2 / 3$ efficiency. The photoelectron energy is sampled using the subshell ionization probabilities and subshell energy levels, and the angle - from the Sauter distribution [4] for the $K$-shell, and from the Gavrila distribution [5] for the $L$ - and $M$-shells. The fluorescence proba- 
bility is estimated from EPDL and the photon is sampled if its energy is above the transport threshold, otherwise it is deposited locally. Secondary energies in $e^{+} e^{-}$ pair production are sampled in accordance with the Tsai approximation [6], with the Thomas-Fermi approximation for the elastic and inelastic screening functions for elements with $Z>5$ and special screening functions for lighter elements. The pair angles are sampled as in [7].

\section{Electron and Positron Physics}

Total $d E / d x_{\text {tot }}$ and restricted $d E / d x_{<}$ionization stopping powers for electrons and positrons are calculated in accordance with ICRU-37 [8]. Ionization potentials and density corrections are included for all 100 elements. Special care is taken for gaseous mixtures. Due to the difference in the scattering laws-Möller for electrons and Bhabha for positrons- $d E / d x$ is calculated separately for $e^{-}$and $e^{+}$. The stopping power above the threshold $\left(d E / d x_{t o t}-d E / d x_{<}\right)$is calculated in the assumption of scattering on free electrons and is expressed as an integral over the analytical Möller or Bhabha cross-sections from the threshold to the maximum energy transfer allowed.

The technique of Ref. [9] is employed for charged particle tracking without simulating the enormous number of low energy transfer collisions. For ionization collisions and bremsstrahlung, a threshold energy transfer $Q_{t}$ is introduced. The processes with energy transfer $Q>Q_{t}$-catastrophic events-are simulated individually. The collisions with $Q \leq Q_{t}$ are lumped together and treated in a continuous way. Two quantities are calculated at the initialization stage: $d E / d x_{<}$and cross-sections above the threshold $\Sigma_{>}$(to select a step length). A combination of $\Sigma_{>}, d E / d x_{<}$and continuous slow down approximation (CSDA) allows one to sample the energy $E$ at the end of the step from a relation

$$
-\log \zeta=\int_{E}^{E_{0}}\left[\Sigma_{>}(E) / d E / d x_{<}(E)\right] d E
$$

where $\zeta$ is a uniform random number of $(0,1)$. A step size is sampled from the CSDA tables. After the step, the phase coordinates due to energy straggling and multiple scattering are sampled.

Multiple Coulomb scattering is simulated from a modified Moliere theory. For the fast sampling, one uses a 2-D table for the inverse probability function to sample $\bar{\theta}^{2}(\zeta, B)$ directly. The straggling distribution function due to ionization collisions with $Q<Q_{t}$ is derived from the Vavilov distribution. The Rutherford part of the scattering cross-section is used, thus one deals with a generic probability distribution function applicable both for $e^{-}$and $e^{+}$. Again, a 2-D table of the inverse probability function is pre-calculated and used in this sampling. The probability of a particular catastrophic event together with the element number are sampled the same way as 
for photons. The $e^{-}$and $e^{+}$elastic scattering is described in a one-photon approximation using the Möller and Bhabha cross-sections. Sampling is quite similar, due to the common Rutherford parts. Energy transfer-and hence scattering angle-is sampled from the Rutherford part and then is rejected or accepted, in accordance with the rest of the cross-section.

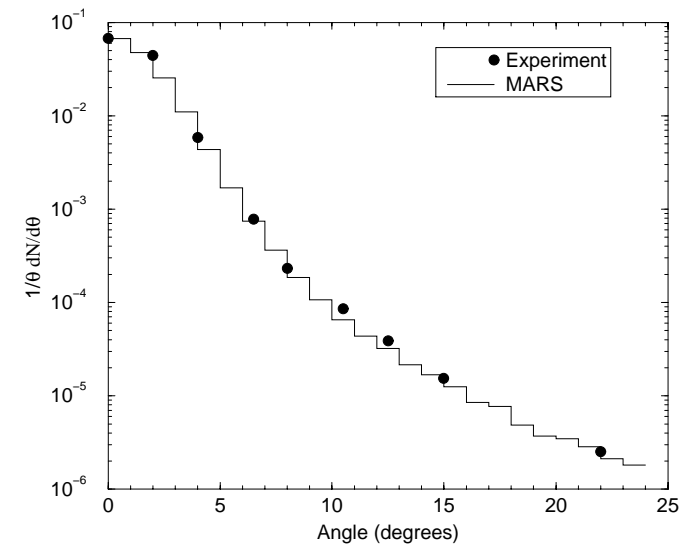

Figure 1: $15.7 \mathrm{MeV} e^{-}$angular distribution after a $18.66 \mathrm{mg} / \mathrm{cm}^{2}$ gold foil, data [11]
Bremsstrahlung data is taken from the tables [10] for differential crosssections $\beta^{2} / Z^{2} \cdot \omega d \sigma / d \omega$ for all 100 elements. The tables are for electrons with kinetic energies from $1 \mathrm{keV}$ to $10 \mathrm{GeV}$. At $E>10 \mathrm{GeV}$, the Tsai analytical expression [6] is used for the differential cross-section with the same approximation for screening functions as used for $e^{+} e^{-}$pair production. The restricted bremsstrahlung stopping power and bremsstrahlung cross-section above the threshold are calculated using numerical integration over the tables [10]. Total and differential cross-sections for positron in-flight annihilation are taken from a one-photon exchange calculation [4]. Sampling is quite simple using a combination of the exact method and a von Neumann rejection. Figs. 1 and 2 show two examples of comparison of MARS calculations with the above algorithms and experimental data $[11,12]$.
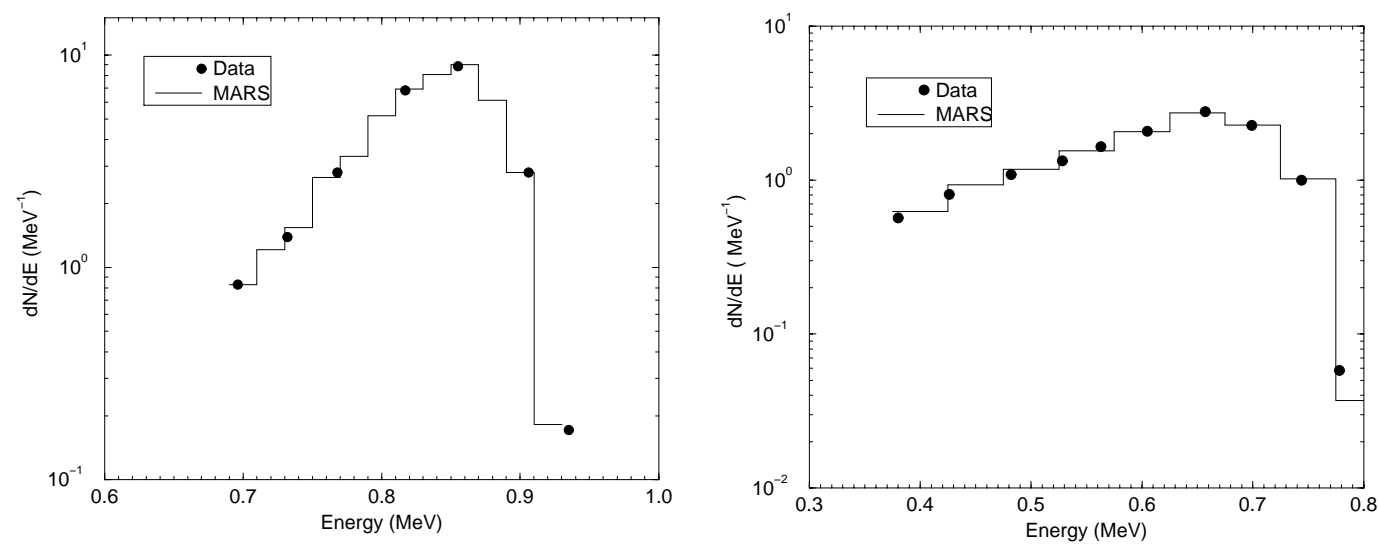

Figure 2: $1 \mathrm{MeV} e^{-}$energy spectra after 0.36 (left) and $0.8 \mathrm{~mm}$ (right) Al foils, data [12] 


\section{Muons, Photohadrons and Polarization}

$e^{+} e^{-} \rightarrow \mu^{+} \mu^{-}$. A sampling algorithm for muon phase coordinates is based on a covariant method for a differential cross-section in a one-photon exchange approximation [4]

$$
\frac{d \sigma}{d s}=\frac{4 \pi e^{4}}{t\left(t-4 m^{2}\right)} \frac{1}{t^{2}}\left\{\frac{s^{2}+u^{2}}{2}+\left(m^{2}+\mu^{2}\right)\left(2 t-m^{2}-\mu^{2}\right)\right\} .
$$

To enhance a muon pair production, MARS uses a forced $e^{+} e^{-}$to $\mu^{+} \mu^{-}$conversion with a probability governed by the user.

$\gamma Z \rightarrow \mu^{+} \mu^{-}$. An exact expression for $\frac{d \sigma}{d \varepsilon_{\mu}}$ is derived for nuclei using a dipole formfactor approximation. It is used for total cross-section numerical calculations and for a muon energy sampling via the von Neumann method. By default, this process is also forced with a production probability controlled by the user.

Photohadrons. Hadroproduction in photon-nucleus interactions is simulated as described in Ref. [1,13], with the analog or forced mode chosen by the user.

Low energy polarized photons. Generic polarized photon transport algorithms developed in [14] are implemented into MARS. A generic particle ID is introduced as a new MARS feature, which allows one to bind the Stokes parameters and local coordinate axis to a low energy photon ID. Fig. 3 gives an example of polarized photon calculations.
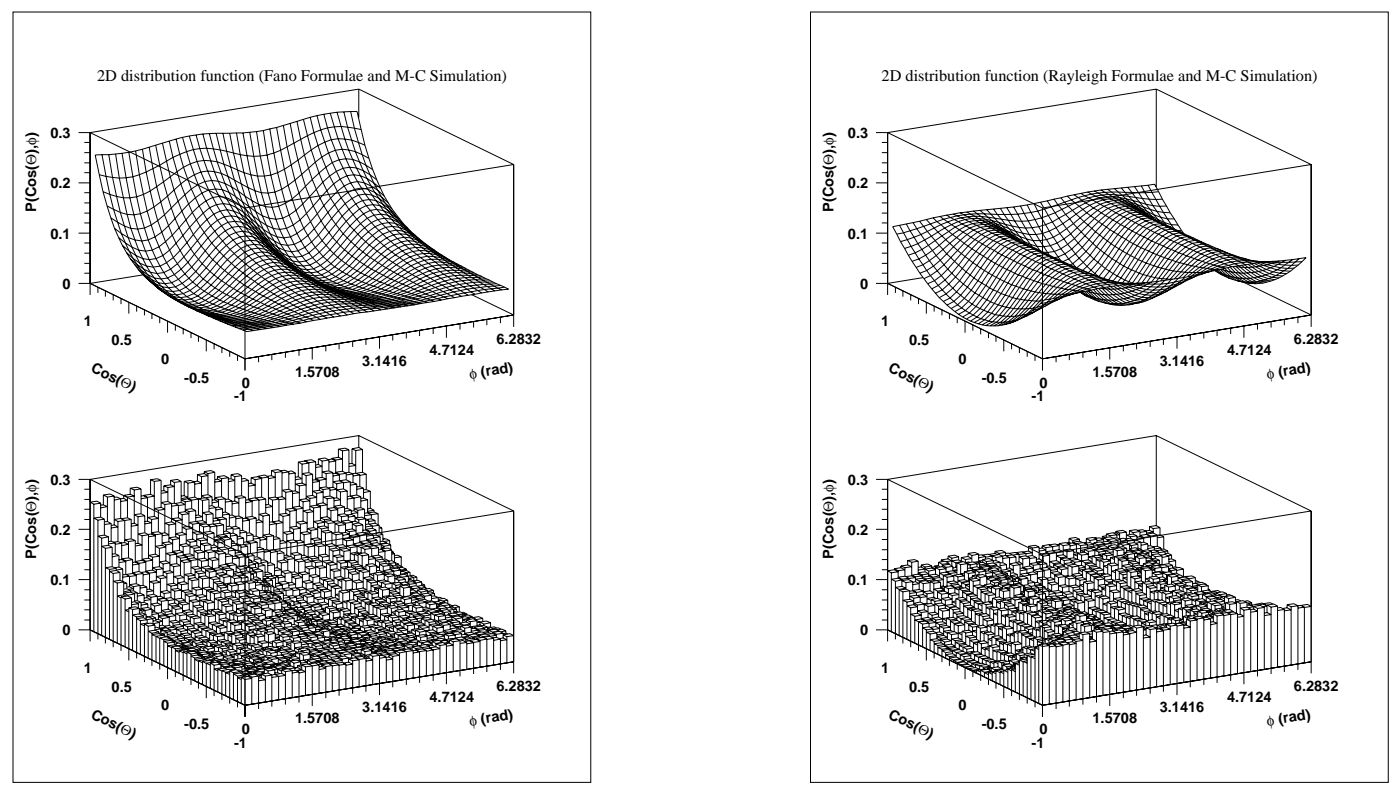

Figure 3: $10 \mathrm{keV}$ polarized photon angular distribution after Compton scattering, incoherent (left) and coherent (right) 


\section{$5 \quad$ EMS Vertex Biasing}

The original MARS EMS module uses a leading particle biasing algorithm (LPBA) [2]. At each vertex, just one particle is picked up for further tracking, another particle is killed. With the probability $E_{\text {out }} / E_{\text {in }}$ a secondary particle with energy $E_{\text {out }}$ is selected and with the probability $1-E_{\text {out }} / E_{\text {in }}$ it is rejected, and the other one is picked up. A statistical weight is adjusted correspondingly, $w_{\text {out }}=w_{\text {in }} E_{\text {in }} / E_{\text {out }}$, to fulfill energy conservation in the event. Such an approach obviously favors a more energetic particle which is appropriate for a deep penetration problem. This algorithm is also present in the new EMS module, as well as a fully analog simulation. Another inclusive algorithm is also introduced, with secondary particle rejection/selection with an equal probability. The choice of one of these three algorithms is controlled by the user.

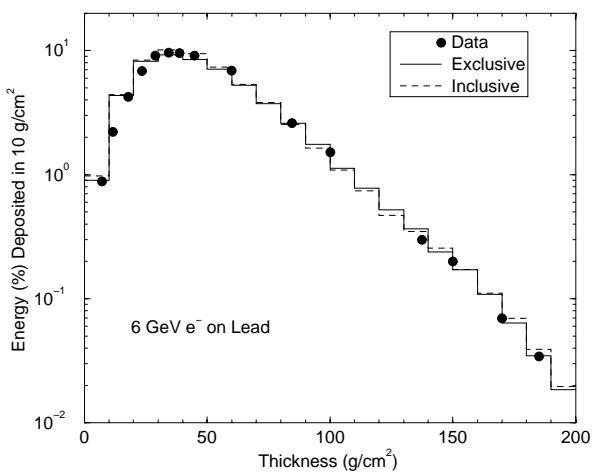

Figure 4: $6 \mathrm{GeV} e^{-}$energy deposition in $\mathrm{Pb}$, as calculated with MARS exclusive algorithm and LPBA $v s$ data [15]

The analog algorithm, allowing one to calculate cascade-by-cascade fluctuations, consumes significant CPU-time per event-which grows linearly with the EMS energy. All three algorithms give similar results for inclusive type distributions, such as energy deposition as shown in Fig. 4 for a $6 \mathrm{GeV} e^{-}$on lead. However, computational efficiencies $\varepsilon=$ $\left(t \sigma^{2}\right)^{-1}$ are different, where $t$ and $\sigma$ are the CPU-time and RMS statistical error, both per event. In the above $6-\mathrm{GeV}$ example, at two representative locationsthe shower maximum and a maximum thickness of $200 \mathrm{~g} / \mathrm{cm}^{2}$ - the corresponding values of $\varepsilon$ are 2190 and 6.3 in the original MARS LPBA with $E_{t h}=100 \mathrm{keV}$.

In the new code with $E_{t h}=1 \mathrm{keV}, t$ is noticeably longer, resulting in lower values of $\varepsilon$ which are now 952 and 2.1 using the analog algorithm and 817 and 3.6 for LPBA. For averaged EMS characteristics, such as energy deposition, LPBA gives better efficiency at large distances compared to the analog algorithm.

\section{Computing issues}

The new EMS modules are written in $\mathrm{C}$, with proper interface to the Fortran parts of the code. An early decision was to embed all data necessary in the course of calculations via static arrays. Therefore there is no need for any additional data files and a file managing code. The price for that decision is the increase in the size of the code of about $20 \mathrm{Mb}$. 
This work was supported by the U.S. Department of Energy. We thank A.A. Wehmann for useful comments.

\section{References}

[1] N.V. Mokhov: 'The MARS Code System User's Guide', Fermilab-FN-628 (1995); O.E. Krivosheev and N.V. Mokhov, 'New MARS and its Applications', in: Proc. of the Third Workshop on Simulating Accelerator Radiation Environments (SARE3), Tsukuba, Japan, May 7-9, 1997, KEK Proceedings 97-5, pp. 12-20 (1997), Fermilab-Conf-98/043 (1998); N.V. Mokhov, S.I. Striganov, A. Van Ginneken, S.G. Mashnik, A.J. Sierk, J.Ranft: in: Proc. of the Fourth Workshop on Simulating Accelerator Radiation Environments (SARE-4), Knoxville, TN, September 14-16, 1998, pp. 87-99, Fermilab-Conf98/379 (1998), nucl-th/9812038-v2-16-Dec-1998; N.V. Mokhov: in Proc. of ICRS-9 International Conference on Radiation Shielding, Tsukuba, Ibaraki, Japan, October 17-22, 1999, J. Nucl. Sci. Tech. 1, pp. 167-171 (March 2000); Fermilab-Conf-00/066 (2000); http://www-ap.fnal.gov/MARS/

[2] A. Van Ginneken: 'AEGIS - a Program to Calculate the Average Behavior of Electromagnetic Showers’, Fermilab-FN-309 (1978)

[3] D.E. Cullen et al.: 'Tables of Graphs of Photon-interaction Cross-Section from $10 \mathrm{eV}$ to $100 \mathrm{GeV}$ Derived from the LLNL Evaluated Photon Data Library (EPDL)', UCRL-50400, Vol. 6, Rev. 4, Oct. 31, LLNL (1989)

[4] L.D. Landau, E.M. Lifshitz: Theoretical Physics. Quantum Electrodynamics, Vol. 4 (Moscow, Nauka 1984)

[5] M. Gavrila: Phys. Rev. 124, 578 (1961)

[6] Y. Tsai: Rev. Mod. Phys. 46, 815 (1974)

[7] A. Borselino: Nuovo Cimento 4, 112 (1947)

[8] ICRU Report 37: Stopping Powers for Electrons and Positrons (Bethesda) MD 20814 (1984)

[9] A.M. Kolchuzhkin et al.: 'Monte Carlo Code for Simulation of ElectronPhoton Showers and Pencil Beam Transport Studies', in: Proc. of 8th Intl. Conf. on Radiation Shielding, Arlington, TX, April 24-28, 1994 pp. 13461350

[10] S.M. Seltser, M.J. Berger: Nucl. Instr. Methods B12, 95 (1985)

[11] A.O. Hanson: Phys. Rev. 81, 634 (1951) 
[12] H.D. Rester et al.: J. Appl. Phys. 41, 714 (1971)

[13] N.V. Mokhov, S.I. Striganov: in Handbook of Accelerator Physics and Engineering. Ed. by A.W. Chao, M. Tigner (World Scientific, Singapore 1999) pp. 234-240

[14] A.M. Kolchuzhkin, O.E. Krivosheev: 'Monte Carlo Simulation of Polarized Photon Transport', in: Proc. of ANS Radiation Protection and Shielding Topical Meeting, Falmouth, MA, April 21-25, 1996, pp. 787-794

[15] G. Bathow et al.: 'Measurements of the Longitudinal and Lateral Development of Electromagnetic Cascades in Lead, Copper and Aluminum at $6 \mathrm{GeV}$ ', DESY-69/39 (1969) 